\title{
Antenna with optimized pattern for simultaneous reception of terrestrial signals and signals of geostationary satellites
}

\author{
G. Saala, D. Müller, J. Hopf, and S. Lindenmeier \\ Institut für Hoch- und Höchstfrequenztechnik, Universität der Bundeswehr München, Werner-Heisenberg-Weg 39, \\ 85577 Neubiberg, Germany
}

\begin{abstract}
The link margin for satellite communication services targeting mobile users is tight. This leads to reception problems in areas with strong fading scenarios like urban areas or forested areas with dense foliage. In order to avoid loss of service in urban areas with week coverage by satellite signals, terrestrial repeaters are used. Therefore in the design of antennas for mobile reception of satellite services, an optimized characteristics for the satellite constellation, as well as an adequate coverage of the terrestrial repeaters has to be accounted for. In this paper an antenna with optimum shaped pattern for the satellite mode and adjustable gain for vertical polarization is investigated. Beside constraints regarding the radiation pattern, measurements concerning the dependence of the antenna on noise temperature and therefore the behavior in real noisy environments are carried out. A comparison with a conventional antenna shows the high performance of the new antenna in combination with a very low volume requirement.
\end{abstract}

\section{Introduction}

Services in satellite communications are not only set up for stationary receiver stations any more. During the last decade more and more services are targeting also mobile users not only for telephony but also for broadcasting radio and data services. Satellite systems such as Satellite Digital Audio Radio Services (SDARS) in the USA and Worldspace in Africa, Europe and parts of Asia, are already in operation. Aspects of the coverage of Europe by Worldspace are examined in Sallam et al. (2008).

For each service a different constellation of satellites might be reasonable, depending on area of coverage, data

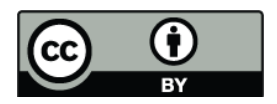

Correspondence to: G. Saala

(gerd.saala@unibw.de)

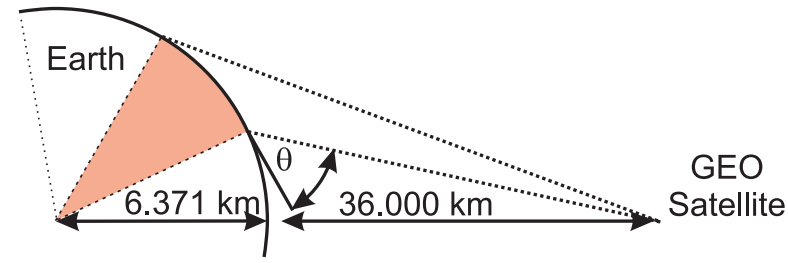

Fig. 1. Sketch of the geostationary satellite constellation for 2-Dapproximation of the elevation angle above the horizon, the satellite is seen under from an earth station.

rates and available transmission power of the satellite. For a wide area of coverage the geostationary orbit (GEO) is chosen for several systems, like Worldspace and XM-SDARS, whereas other systems operate in high earth orbits (HEO), such as Sirius-SDARS which is described in Briskman and Prevaux (2004) using several satellites to realize the continuous coverage of the service. An overview of several satellite services is given in Lida et al. (2003).

For a coverage of the USA and Europe the minimum latitude is approximately $\mathrm{Lat}_{\min } \approx 26^{\circ} \mathrm{N}$ for the southern point of Texas. The maximum latitude for the north of Denmark is approximately Lat $\mathrm{Lax}_{\max } \approx 57^{\circ} \mathrm{N}$. From Fig. 1, with an easy two dimensional approximation it can be seen, that geostationary satellites in these areas of Europe and the USA are seen in an elevation angle from $25^{\circ}$ to $60^{\circ}$ (Morgan and Gordon, 1989). In the following antennas are investigated which are designed for services using geostationary satellites. As the satellites are never seen at zenith or the horizon, respectively, the radiation pattern should be low at these positions for optimum power distribution in ranges of the elevation angles the satellites may appear in. In contrast to stationary receiving stations where the antenna beam can always point towards the satellite in a fixed direction, the azimuth angle under which the satellite occurs is unknown in mobile

Published by Copernicus Publications on behalf of the URSI Landesausschuss in der Bundesrepublik Deutschland e.V. 


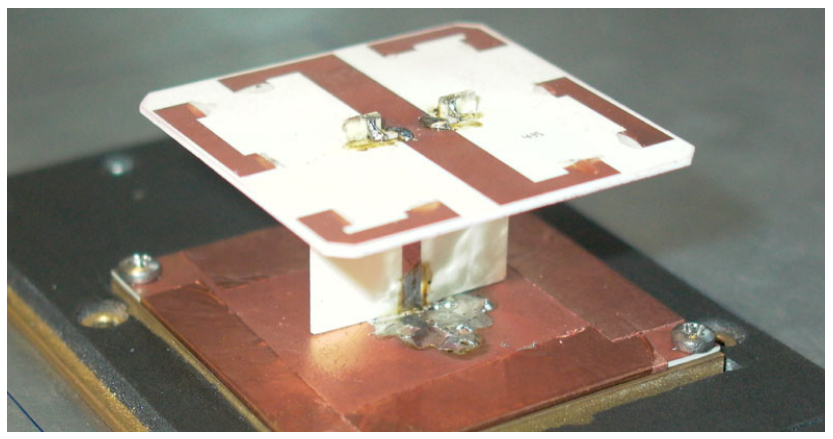

Fig. 2. Complete antenna structure with vertical feed section realized as double sided parallel strip line.

applications. Hence, the radiation pattern should be omnidirectional over the azimuth. A further constraint arises from the sufficient coverage of areas with strong fading scenarios or weak signals like urban areas. In those areas the coverage is supported by additional terrestrial repeaters which transmit in vertical polarization. In this paper, an antenna with an adjustable power ratio between vertical and horizontal polarization is investigated. Considering the vertical polarization with respect to the performance of the reception of satellite signals, also the noise behavior of the antenna with better terrestrial reception is analyzed. Finally field tests are presented which compare the performance in signal to noise ratio (SNR) of the new antenna type with a commercially available antenna with respect to different receiving environments.

\section{Antenna structure}

The principle of a new antenna structure has been presented in Saala et al. (2009). It consists of an electrically large loop structure over a ground plane. This loop structure is segmented in order to achieve a homogeneous current distribution over the complete circumference of the horizontal part of the antenna. With that homogeneous current over the ground plane the main beam is directed to an elevation angle of approximately $\theta \approx 45^{\circ}$ and a null at the zenith and horizon. In the following several variants of this antenna, shown in Fig. 2, are investigated with respect to the adjustment of the vertical polarized part of the radiation pattern. The antennas are designed for a center frequency of $f_{\mathrm{c}}=1.486 \mathrm{GHz}$.

\subsection{Basic operation mode}

The basic antenna consists of the horizontal loop structure which is fed by horizontal double sided parallel strip line connected to a vertically oriented feed section, e.g. coaxial line. By proper design an additional matching network can be avoided at the base point of the coaxial line. The vertical part can also be realized by a double sided parallel strip

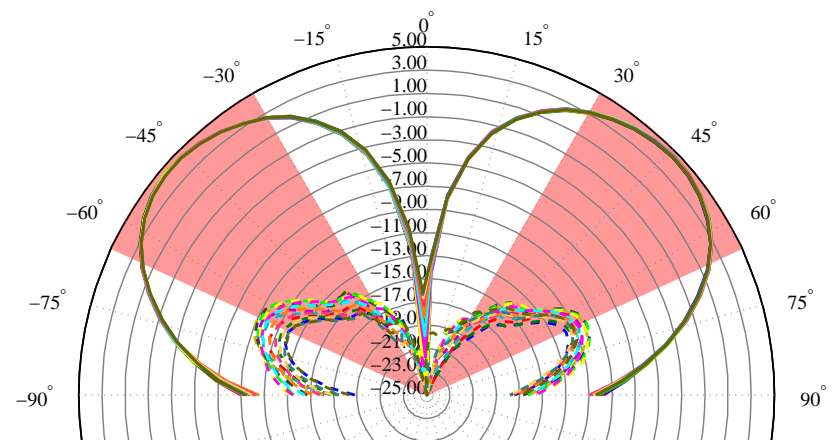

Fig. 3. Basic antenna setup with coaxial feed. 16 elevation cuts for vertical (- - ) and horizontal (-) polarization in azimuthal steps of $\Delta \varphi=11.25^{\circ}$.

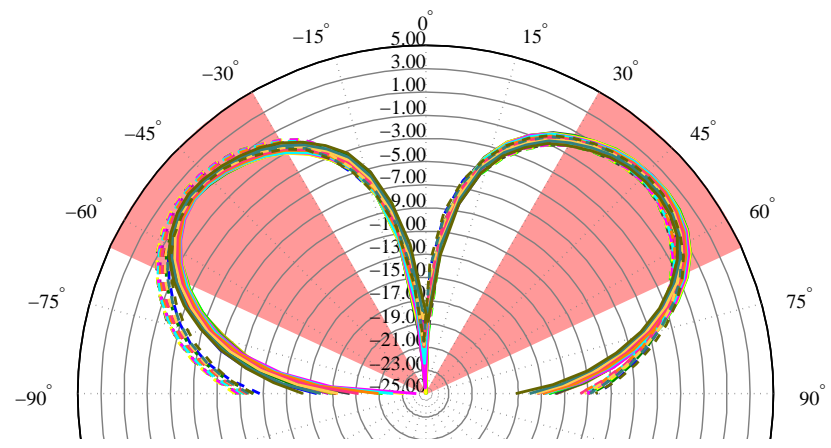

Fig. 4. Basic antenna setup with coaxial feed. 16 elevation cuts for right (-) and left $(--)$ hand circular polarization in azimuthal steps of $\Delta \varphi=11.25^{\circ}$.

line for easier production. In Figs. 3 and 4 the radiation patterns are depicted for linear and circular polarizations, respectively. The horizontal polarization is perfectly rotational symmetric over the azimuth. The vertical polarization is more than $15 \mathrm{~dB}$ lower than the horizontal polarization. The circular polarizations are also equally balanced with a little asymmetry over the azimuth, which is due to the asymmetry of the vertical polarization caused e.g. by effects of the antenna chassis. With this antenna a simultaneous reception of data streams transmitted in right and left hand polarization is possible without necessity of a switch, which might interrupt the data stream when switching from one polarization towards the other one.

\subsection{Stronger terrestrial mode}

For optimum coverage not only via satellites but also by terrestrial repeaters, the gain in vertical polarization of the antenna has to be adjusted to the needs of the service. Therefore the loop structure of the antenna is enhanced to perform in a mixed mode operation by introducing a capacitor at the base 


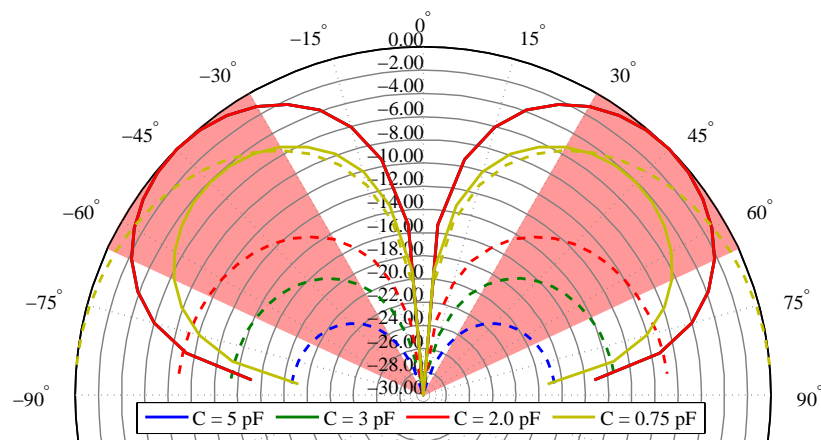

Fig. 5. Simulation: Different values of capacitance introduced at the feed point of the antenna. Vertical (--) and horizontal (-) polarized radiation pattern, the curves are average values over the azimuth.

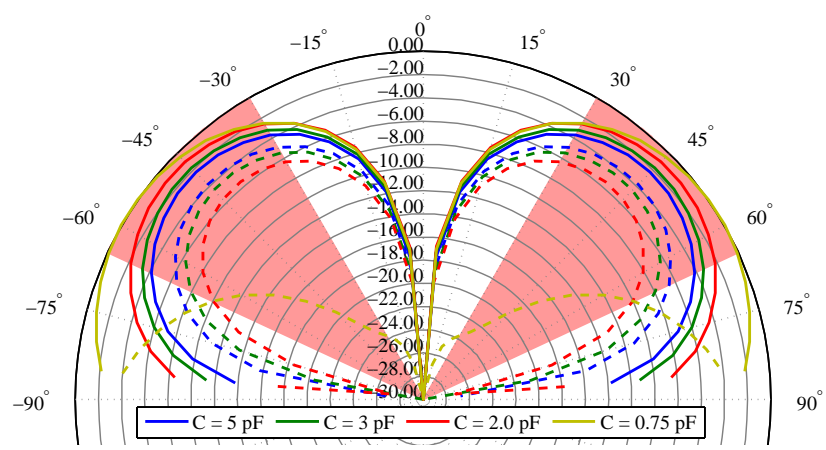

Fig. 6. Simulation: Different values of capacitance introduced at the feed point of the antenna. Right (-) and left $(--)$ hand circular polarized radiation pattern, the curves are average values over the azimuth.

point of the vertical feed section of the antenna. With this capacitor a common mode current is excited on the vertical section, which leads to a raised radiation in vertical polarization.

Compared to the antenna operating in its basic mode with mainly horizontal polarized radiation, the introduction of a capacitor changes the input impedance of the antenna, which cannot easily be accounted for by change of the loopstructure or the feed line. Hence an additional matching network is required. The power ratio between the horizontal and vertical polarization is not influenced by this matching network, compared to the unmatched operation.

Figures 5 and 6 show simulation results of antennas with different values of capacitors introduced at the feed point of the antenna. In order to show the tendency of the power ratio independent of the matching, the graphs are normalized values of each antenna. For the linear polarizations a fine graduation over the capacitance is achieved. For very small capacitors the vertical polarization even exceeds the hori-

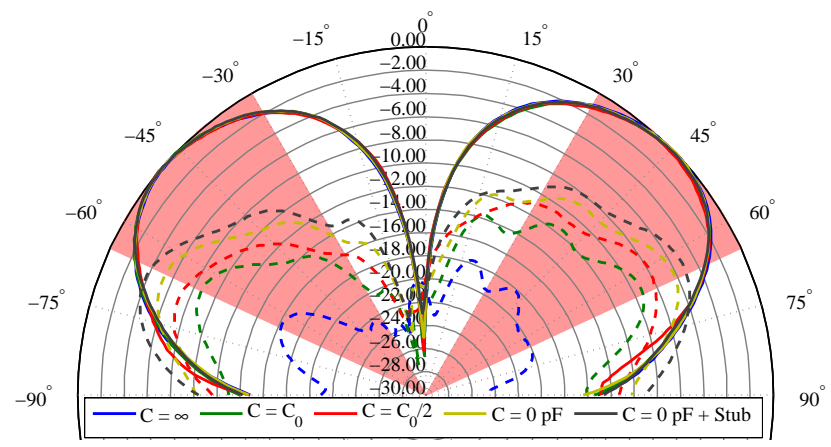

Fig. 7. Measurement: Vertical (--) and horizontal (-) polarized radiation pattern for different capacitances at the feed point. Each curve is the average over four equally spaced elevation cuts with $\Delta \varphi=45^{\circ}$.

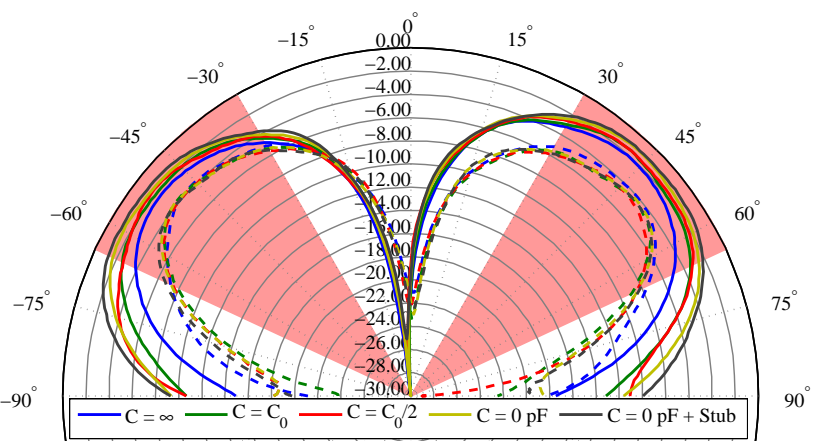

Fig. 8. Measurement: Right (-) and left (--) hand circular polarized radiation pattern for different capacitances at the feed point. Each curve is the average over four equally spaced elevation cuts with $\Delta \varphi=45^{\circ}$.

zontal polarization. Because of the excitation of the common mode current at the base point of the antenna the phase for the received signals is not necessarily the same for vertical and horizontal polarization. This leads to an imbalance between the circular polarizations (Fig. 6) which gets greater with rising vertical polarization. This imbalance can be used to account for an imbalance of the power distribution to the two circular polarizations on the satellite.

For the measurements, shown in Figs. 7 and 8, the capacitors at the base point of the antenna and the matching network have been introduced on the rear side of the ground plane. The plots for the linear polarizations in Fig. 7 show the precisely adjustable power ratio $H / V$ between the horizontal and vertical polarization starting from the basic mode operation for the short with $C_{\text {feed }}=\infty$ and ending with a large reactance for the open circuit with $C_{\text {feed }}=0 \mathrm{pF}$. Compared to the simulations the power ratio $H / V$ is different e.g. in case of $C=0 \mathrm{pF}$. This is due to a stray capacitance between the open conductor and the ground plane. 


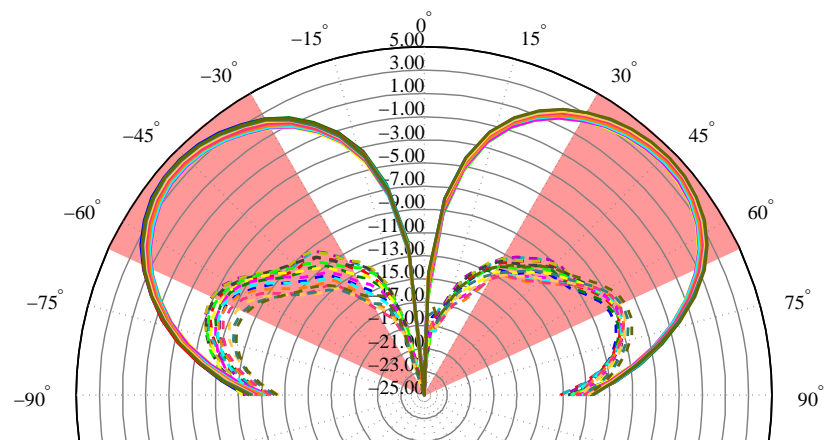

Fig. 9. Capacitor at the base of the vertical feed structure. 16 elevation cuts for vertical (--) and horizontal (-) polarization in azimuthal steps of $\Delta \varphi=11.25^{\circ}$.

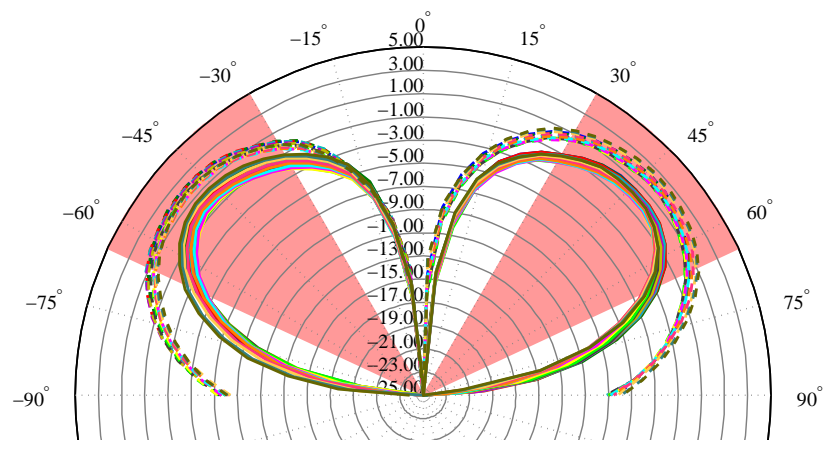

Fig. 10. Capacitor at the base of the vertical feed structure. 16 elevation cuts for right (-) and left (--) hand polarization in azimuthal steps of $\Delta \varphi=11.25^{\circ}$.

The radiation pattern of one antenna sample with increased shape of vertical the polarization, matched to $50 \Omega$ is shown in Fig. 9 for the linear polarization with a power ratio of $H / V \approx 9 \mathrm{~dB}$. The radiation pattern of the circular polarizations in Fig. 10 show an imbalance of up to $2 \mathrm{~dB}$.

\subsection{Suppression of the terrestrial mode}

The increased gain in vertical polarization, described in Sect. 2.2, is due to a common mode current excited at the base of the vertical feed section. A small fraction of a common mode current is also excited using a coaxial line or parallel strip line, respectively for this feed section. For an additional discrimination of the parasitic vertical polarized radiation a balanced unbalanced transition could be introduced at the feed point of the antenna. Another solution is achieved by introducing two capacitors at the top of the vertical feed section. Thereby a common mode current is excited also at the top section of the vertical feed, which interferes destructively with the parasitic common mode current excited at the base point of the antenna. The resulting radiation pattern for this

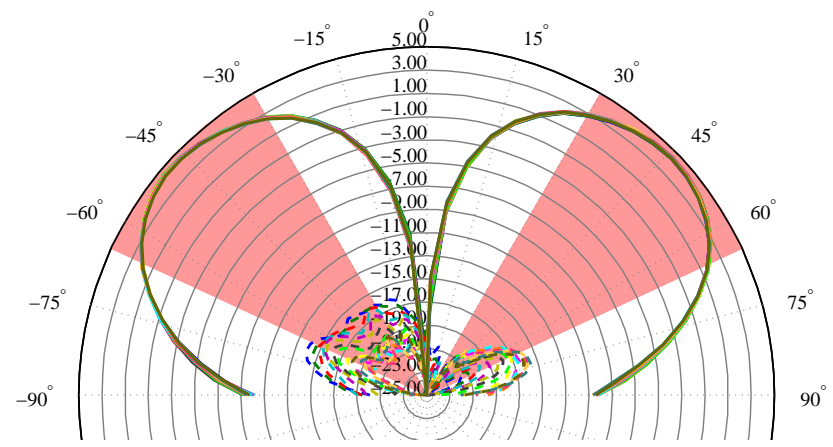

Fig. 11. Capacitors at the top of the vertical feed section. 16 elevation cuts for vertical (--) and horizontal (-) polarization in azimuthal steps of $\Delta \varphi=11.25^{\circ}$.

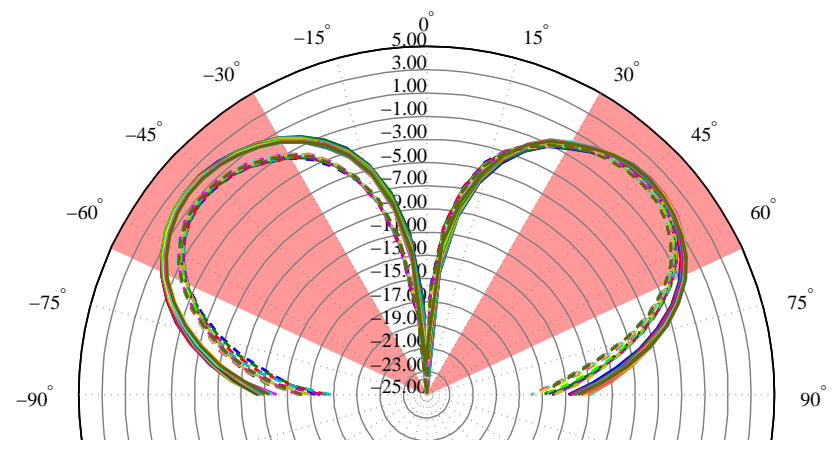

Fig. 12. Capacitors at the top of the vertical feed section. 16 elevation cuts for right (-) and left $(--)$ hand polarization in azimuthal steps of $\Delta \varphi=11.25^{\circ}$.

antenna is shown in Fig. 11 for the linear polarizations with a power ratio of $H / V \approx 18.5 \mathrm{~dB}$. The left hand and right hand circular polarizations in Fig. 12 are almost balanced.

A comparison of the phases of the received signals of the antennas described in the last sections is shown in Fig. 13. Only for the basic mode the signals for vertical and horizontal polarization are in phase or anti-phase respectively, which is the reason for the very good balance between left and right hand circular polarizations in this case.

\section{Noise measurements and antenna temperature}

The system noise temperature $T_{\mathrm{S}}$ at the antenna terminals considering antenna losses, attenuation on the transmission line and noise of the amplifiers or receiver respectively, which is used to calculate the $G / T$ of a satellite receiving antenna, is given by Kraus and Marhefka (2003) (Eq. 1).

$$
T_{S}=T_{\mathrm{A}, \mathrm{rad}}+T_{0}\left(\frac{1}{\eta_{\mathrm{A}}}-1\right)+T_{0}\left(\frac{1}{\eta_{\mathrm{L}}}-1\right)+\frac{T_{\mathrm{R}}}{\eta_{\mathrm{L}}}
$$




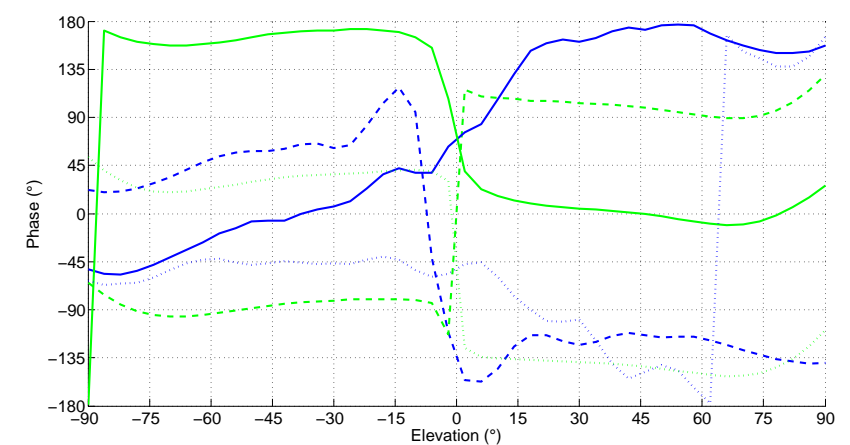

Fig. 13. Transmission phases for vertical (blue) and horizontal (green) polarizations; antenna in basic (-), stronger vertical (--) and suppressed vertical polarization $(\cdots)$ mode.

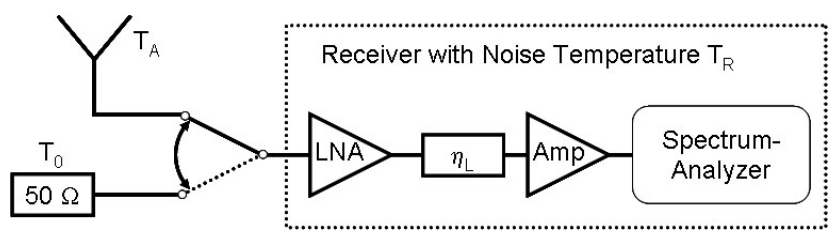

Fig. 14. Measurement setup for determination of the antenna noise temperture.

In order to investigate the impact of the surrounding temperature on the noise characteristics of antennas with varied adjustment of the radiation pattern the three antennas described in Sects. 2.1, 2.2 and 2.3 are compared in noise measurements with respect to the radiation part of the antenna noise temperature $T_{\mathrm{A}, \text { rad }}$.

Each of the three antennas are matched to the system impedance of $Z_{S}=50 \Omega$. In the noise measurement each antenna is compared to the temperature of a reference resistor with a temperature of $T_{\mathrm{Ref}}=T_{0}=293 \mathrm{~K}$, using the measurement setup depicted in Fig. 14. The noise temperature of the low noise amplifier (LNA), the transmission line and the power amplifier are summarized in the receiver temperature $T_{\mathrm{R}}$ at the input of the LNA. The Antenna Temperature $T_{\mathrm{A} \text {,rad }}$ has to be determined. With the reference resistor switched to the low noise amplifier (LNA) the system noise temperature at the input of the receiver is given from Eq. (2). Equation (3) determines the measured noise temperature with the antenna under test switched to the receiver.

$$
\begin{aligned}
T_{\mathrm{S}, \text { Ref }} & =T_{0}+T_{\mathrm{R}} \\
T_{\mathrm{S}, \mathrm{A}} & =T_{\mathrm{A}, \mathrm{rad}} \cdot \eta_{\mathrm{A}}+T_{\mathrm{A}, \text { Loss }}+T_{\mathrm{R}},
\end{aligned}
$$

where $T_{\mathrm{A}, \text { Loss }}=T_{0} \cdot\left(1-\eta_{\mathrm{A}}\right)$ is the noise temperature contributed by the losses in the antenna. The efficiency of the antenna is determined by calculations from gain measurements of the radiation pattern. Therefore the measured directional characteristic of the antenna is compared with an

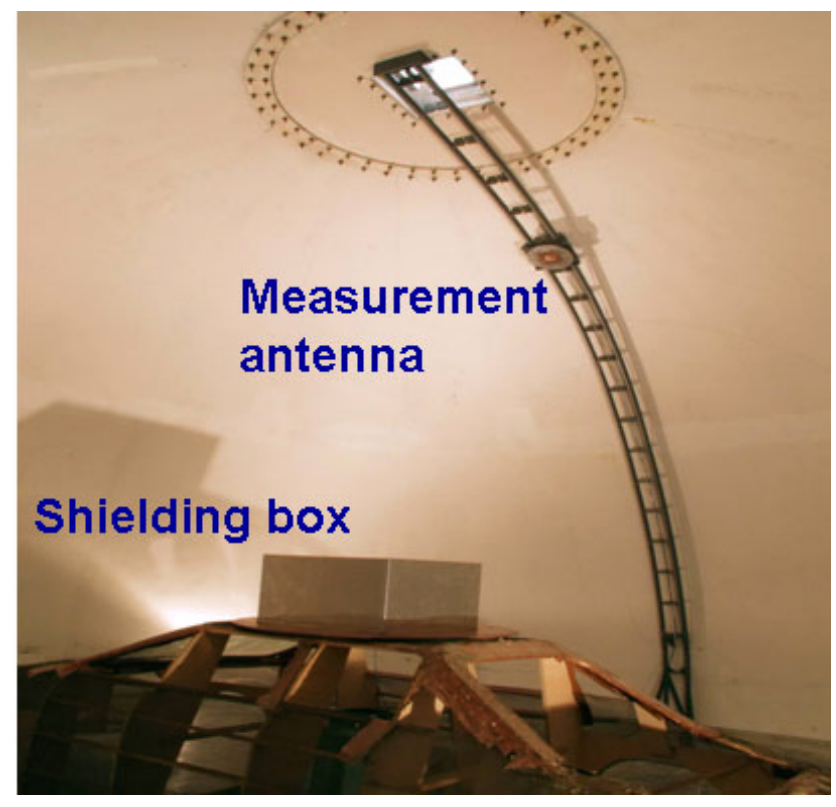

Fig. 15. Facility for antenna noise temperature $T_{\mathrm{A}}$ measurements.

ideal isotropic radiator at the same sampling points as in the measurement. With the power ratio

$\Delta P_{\mathrm{S}}=\frac{P_{\mathrm{S}, \mathrm{A}}}{P_{\mathrm{S}, \text { Ref }}}=\frac{T_{\mathrm{S}, \mathrm{A}}}{T_{\mathrm{S}, \text { Ref }}}$

the radiation part of the antenna temperature $T_{\mathrm{A}, \text { rad }}$ can be determined to

$T_{\mathrm{A}, \mathrm{rad}}=\frac{1}{\eta_{\mathrm{A}}} \cdot\left[\Delta P_{\mathrm{S}} \cdot\left(T_{0}+T_{\mathrm{R}}\right)-T_{\mathrm{R}}-T_{\mathrm{A}, \text { loss }}\right]$

In the presented measurements the radiation part of the antenna temperature is investigated in dependence of the radiation characteristics, especially the power ratio between horizontal and vertical polarization. The measurements have been performed in a $T_{\mathrm{A}}$ measurement facility shown in Fig. 15 which is covered by a radom for weather protection. The antenna unter test is positioned on a car model. Using the measurement antenna, which can be positioned in elevation, also $G / T$-mesurements can be performed. The results of the measurements are shown in Table 1 . The difference within $\Delta f=2 \mathrm{MHz}$ is, beside a slight change of the antenna impedances, mainly caused by the edge of the transmitted signal being close to the center frequency. For the two antennas with lower vertical polarization the results are similar. A significant increase of the antenna temperature occurs for the antenna with stronger vertical polarization. The difference in antenna temperature is comparable with the noise contributed by losses in the antenna of more than $0.2 \mathrm{~dB}$ to $0.3 \mathrm{~dB}$. Hence, considering noise aspects the vertical polarized radiation of an antenna should be kept as low as possible for satellite applications. Overall the new antenna shows very good results in noise temperature. 
Table 1. Results of noise measurements of three antennas with different power ratio $(H / V)$ of vertical and horizontal polarization with center frequency $f_{\mathrm{c}}$.

\begin{tabular}{llll}
\hline Antenna & $H / V$-ratio & $\begin{array}{l}T_{\mathrm{A}, \text { rad }} @ \\
f_{\mathrm{c}}\end{array}$ & $\begin{array}{l}T_{\mathrm{A}, \mathrm{rad}} @ \\
f_{\mathrm{c}}+2 \mathrm{MHz}\end{array}$ \\
\hline Reduced terr. pol. & $18.5 \mathrm{~dB}$ & $28.6 \mathrm{~K}$ & $16.0 \mathrm{~K}$ \\
Basic terr. pol. & $15.0 \mathrm{~dB}$ & $31.1 \mathrm{~K}$ & $13.3 \mathrm{~K}$ \\
Increased terr. pol. & $9.0 \mathrm{~dB}$ & $57.8 \mathrm{~K}$ & $36.5 \mathrm{~K}$ \\
$T_{\mathrm{A}, \text { loss }} @$ & $\mathrm{n} / \mathrm{a}$ & $13.2 \mathrm{~K}$ & $13.2 \mathrm{~K}$ \\
$\eta=-0.2 \mathrm{~dB}$ & & & \\
\hline
\end{tabular}

\section{Results of field-tests}

For performance tests in real receiving scenarios the new antenna has been tested in comparison with a conventional antenna which can be switched between left and right hand circular polarization. The optimum polarization matching due to switching is achieved by a seriously higher effort in the amplifier network. Both antennas have been placed in similar positions on a car in three different scenarios. Hardware effort and volume requirement of the new antenna are less than half the one of the reference antenna. At the open range no surrounding objects have been present. In the second scenario the car has been positioned at the edge of a forest with moderate shading of the satellite signals by the trees. In the third case the test position has been inside a forest with shading by high trees. The signals for the circular polarizations have been imbalanced at the satellite with lower power for LHCP. The results in Table 2 show a comparable performance for the new antenna. With only half the hardware effort and volume requirement, in noisy environments like the forest it even outperforms the reference antenna.

\section{Conclusions}

A new antenna for mobile reception of signals transmitted via geostationary satellites is presented. With an optimum shaping of the radiation pattern it is possible to receive signals of left and right hand polarization simultaneously. Therefore disruption of the signal due to switching is avoided. For the coverage of terrestrial repeaters in urban areas, a method for an adjustable power ratio between vertical and horizontal polarization is presented. Noise measurements show how important a precisely adjustable vertical polarization is for satellite applications. Finally tests in real receiving scenarios have shown a very good performance of the new antenna comparable to a high end switchable reference antenna which needs seriously higher mounting volume and effort in fabrication.
Table 2. Results of field tests; comparison of the signal to noise ratio between commercially available antenna with the new loop type antenna.

\begin{tabular}{llll}
\hline SNR of & $\begin{array}{l}\text { Open } \\
\text { Range }\end{array}$ & $\begin{array}{l}\text { Edge of } \\
\text { Forest }\end{array}$ & $\begin{array}{l}\text { Inside } \\
\text { Forest }\end{array}$ \\
\hline $\begin{array}{l}\text { Ref. Antenna } \\
\text { RHCP }\end{array}$ & $9.74 \mathrm{~dB}$ & $5.77 \mathrm{~dB}$ & $2.60 \mathrm{~dB}$ \\
$\begin{array}{l}\text { New Loop } \\
\text { Antenna RHCP }\end{array}$ & $9.68 \mathrm{~dB}$ & $4.60 \mathrm{~dB}$ & $4.40 \mathrm{~dB}$ \\
$\begin{array}{l}\text { Ref. Antenna } \\
\text { LHCP } \\
\text { New Loop }\end{array}$ & $6.90 \mathrm{~dB}$ & $1.97 \mathrm{~dB}$ & $1.00 \mathrm{~dB}$ \\
Antenna LHCP & $4.43 \mathrm{~dB}$ & $1.60 \mathrm{~dB}$ & $2.30 \mathrm{~dB}$ \\
\hline
\end{tabular}

\section{References}

Briskman, R. D. and Prevaux, R. J.: S-DARS broadcast from inclined, elliptical orbits, Acta Astronaut., 54, 503-518, 2004.

Kraus, J. D. and Marhefka, R. J.: Antennas: for all applications, vol. 3, McGraw-Hill, New York, 2003.

Lida, T., Pelton, J., and Ashford, E.: Satellite Communications in the 21st Century: Trends and Technologies, vol. 202, Prog. Astronaut. Aero., 2003.

Morgan, W. L. and Gordon, G. D.: Communications Satellite Handbook, vol. 1, John Wiley, New York, 1989.

Saala, G., Hopf, J., and Lindenmeier, S.: Small satellite car antenna for simultaneous reception of LHCP and RHCP signals, in: 3rd European Conference on Antennas and Propagation (EuCAP) 2009, vol. 3, 2698-2700, 2009.

Sallam, H., Abdel-Nabi, T., and Soumagne, J.: A GEO satellite System for Broadcast Audio and Multimedia Services Targeting Mobile Users in Europe, in: 4th Advanced Satellite Mobile Systems Conference (ASMS) 2008, vol. 4, 134-139, 2008. 